\title{
Estrategia Pedagógica basada en las Tecnologías de Información y Comunicación dirigida a Padres y/o Cuidadores ${ }^{1}$
}

\section{Pedagogic Strategy based on Information Technology of Communication addressed to Parents and / or Caregivers}

DOI: http://dx.doi.org/10.17981/cultedusoc.9.3.2018.14

Artículo de investigación. Fecha de recepción: 15/06/2018. Fecha de aceptación: 27/11/2018

\author{
Neyla Yancy-Orozco ${ }^{2}$; \\ Jairo Cantillo-Chiquillo; Kelly Correa-Cárdenas; \\ Oscar Nossa-Plata; Clara Vargas -Lobo ${ }^{3}$
}

Institución educativa departamental María Inmaculada del Municipio de Pivijay Magdalena (Colombia) neylayancy@hotmail.com

Para citar este artículo:

Yancy-Orozco, N., Cantillo-Chiquillo, J., Correa-Cárdenas, K., Nossa-Plata, O. y Vargas-Lobo, C. (2018). Estrategia Pedagógica basada en las Tecnologías de Información y Comunicación dirigida a Padres y/o Cuidadores. Cultura. Educación y Sociedad 9(3), 113-122. DOI: http://dx.doi.org/10.17981/cultedusoc.9.3.2018.14

\section{Resumen}

El tema de la familia involucrada en el contexto educativo ha sido una constante discusión abordada desde diferentes áreas del saber, llegando a coincidir en la necesidad de involucrarlos en los procesos de enseñanza, porque así se consolidan en los estudiantes competencias e intereses por la educación. A partir de esto se platea como propósito de esta investigación, fortalecer los procesos educativos a través de las Tecnologías de la información y la comunicación (TIC), involucrando en el proceso de enseñanza aprendizaje a padres de familia y/o cuidadores. Se tomó como unidad de análisis a 20 padres de familias y 9 cuidadores de los estudiantes del quinto grado A $\left(5^{\circ}\right.$ a) y quinto $\mathrm{B}$ $\left(5^{\circ} \mathrm{b}\right)$, de la Institución Educativa Departamental María Inmaculada del Municipio de Pivijay Magdalena, la metodología empleada se orientó desde el enfoque cualitativo, con alcance es descriptivo. Dentro de los hallazgos del estudio se encontró que la mayoría de los padres y/o cuidadores afirman emplear el computador y el internet para ayudar a los estudiantes a realizar sus tareas; que al trabajar de la mano padre y estudiantes, mediados por TIC se fortalecen los procesos pedagógicos y los lazos afectivos entre estos.

Palabras clave: Tecnologías de la información y la comunicación, Estrategia Pedagógica, padres, cuidadores.

\section{Abstract}

The theme of the family involved in the educational context has been a constant discussion addressed from different areas of knowledge, coming to agree that by involving them in the teaching processes, students' competences and interests for education are consolidated. Therefore, the present research is aimed at strengthening educational processes through Information and Communication Technologies (TIC) involving parents and / or caregivers in the teaching-learning process. The unit of analysis was constituted by the students of 5 th $a$ and $b$ of the Departmental Educational Institution María Inmaculada of the Municipality of Pivijay Magdalena. The methodology used was oriented from the Qualitative Approach, with scope is descriptive. Among the findings of the study it was found that most parents and / or caregivers claim to use the computer and the internet to help students perform their tasks; that by working hand in hand with parents and students, mediated by ICT, the pedagogical processes and the affective bonds between them are strengthened.

Keywords: Parents, caregivers, Information Technology of Communication, pedagogical strategy.

\footnotetext{
1 Este artículo ha sido derivado del Programa de Fortalecimiento de la Cultura Ciudadana y Democrática CT+I a través de la IEP apoyada en TIC en el Departamento de Magdalena: proyecto Ciclón.

2 Líder del Grupo de investigación "Los innovadores".

3 Docentes de la institución educativa departamental María Inmaculada del Municipio de Pivijay Magdalena, del grupo de investigación "Los innovadores".
} 


\section{Introducción}

En la actualidad son muchos los cambios que se han venido presentado al interior de las familias, cambios generados por los procesos de transformación social, que se vienen manifestando en cuestiones como: la estructura familiar, la convivencia, las relaciones entre los miembros de la familia, los nuevos sistemas de comunicación e información y tecnológico que influyen en la percepción y conducta de cada uno de sus integrantes (Millán, Gutiérrez y Hernández, 2016).

Según Sánchez (2011), en el tiempo actual debido a la proliferación e impacto de las nuevas tecnologías, todos los aspectos de la vida tanto educativa como social giran en torno al poder, tanto así, que el uso de ellas pasó de ser una herramienta para comunicarse, a convertirse en herramientas para compartir, aprender, indagar y crear espacios de interacción social.

Teniendo en cuenta lo anterior se hace necesario abordar los contendidos del aula, acompañadas de contenidos virtuales que dinamicen el proceso de aprendizaje de los estudiantes, actividades que no solo se limiten al aula de clase, si no que integren también la educación académica y social, que posibiliten la construcción aprendizajes en cualquier momento y lugar a través de herramientas tecnológicas conectadas a la internet (Vargas, 2003).

Partiendo del hecho de que las nuevas tecnologías de la información y la comunicación juegan un papel fundamental en la educación de los hijos, es necesario que docentes y padres trabajen articuladamente en la construcción de saberes, pues estas herramientas influyen directamente en su interés, percepciones e ideas; es aquí donde Martínez, Frías y Solano (2011), sostienen que el internet debido a su rápida proliferación y fácil acceso, se encuentra ligado a los niños y niñas desde temprana edad, pues sus actividades de ocio, entretenimiento y tareas se resuelven mediados por estas; convirtiéndolas en parte de su realidad.
Es importante resaltar que la educación es una responsabilidad compartida entre maestros y padres de familias y/o cuidadores, en este sentido, Kliksberg (2008), sostiene que la familia es el núcleo de la sociedad, la primera escuela donde se forman los valores, los primero aprendizajes, la identidad, y el desarrollo emocional; pues es aquí en este entorno donde los niños y niñas desarrollan su estructuras de personalidad; pues los aprendizajes obtenidos en la familia, contribuyen de manera positiva o negativa a las relaciones sociales fuera de ella.

Debido a esto, el padre de familia o cuidador como ente de construcción de normas, saberes y de trasmisor de conocimiento debe involucrarse en todo proceso académico donde este inmerso su hijo; realizando un debido acompañamiento para el manejo adecuado y precavido del uso de estas herramientas (Betancur, Carmona, Contreras, Karam, Maestre, Romero y Uribe, 2014).

Teniendo en cuenta esta necesidad surge la presente investigación, con el propósito de fortalecer los procesos educativos a través de estrategias pedagógicas basadas en las tecnologías de la información y la comunicación, en adelante denominadas TIC, orientada hacia los padres de familia y/o cuidadores de los estudiantes de $5^{\circ}$ a y b de la Institución Educativa Departamental María Inmaculada del Municipio de Pivijay Magdalena, mediante la implementación de talleres lúdicos-pedagógicos que ofrezcan herramientas para optimizar, fortalecer, impactar e innovar el acompañamiento familiar en los procesos educativos, partiendo de la utilización de las herramientas tecnológicas (Computador, Tablet), que permita adquirir destrezas, habilidades y competencias en los diferentes ámbitos, lo cual conlleva a la participación activa a las actividades lideradas por los diferentes actores que hacen parte de la comunidad educativa. 
A continuación, se presentarán los aspectos conceptuales que se tomaron en cuenta para el desarrollo de la investigación.

\section{La familia y las TIC en el aprendizaje}

Según el Ministerio de Educación Nacional en los últimos años se han incorporado al sector educativo las tecnologías de la comunicación e información en el aula de clase, por lo cual se hace necesario construir estrategias que permitan aprovecharlas no dentro del contexto escolar si no fuera de él; teniendo encuentra esto, es necesario que el padre de familia se involucre en este nuevo escenario, donde se logre formar a un estudiante capaz de responder a cualquier reto presente en la sociedad actual.

Hurtado y De la Hoz (2015), en su proyecto de implementación de estrategias pedagógicas dirigidas a los padres de familia para mejorar el rendimiento escolar de los niños y niñas de la escuela nueva frutier de Santa Catalina (Bolívar), buscaron implementar estrategias pedagógicas a través de talleres que brindaron orientaciones a los padres de familia de la Escuela Nueva Frutier de Santa Catalina (Bolívar), para mejorar el rendimiento escolar; pues su problemática planteada es el bajo rendimiento escolar; el cual era generado en su gran mayoría por la misma ausencia y/o acompañamiento familiar en los procesos educativos de los niños y niñas. Estos encontraron que el problema radicaba en la falta de incentivos por sus padres a la hora de realizar las labores educativas, dando como resultado una afectación en el rendimiento escolar de los niños y niñas. Este resultado nos permite reconocer la importancia del acompañamiento del padre de familia dentro del contexto educativo, permitiendo el fortalecimiento de las diferentes áreas del ser humano; personal, social, familiar y educativo, destacando las habilidades y destrezas que caracte- riza cada estudiante para la obtención de su proyecto de vida. Desde los aportes de Romero y Contreras (2015), se infiere que el desarrollo de capacidades asociadas al proyecto de vida individual implica un proceso de reconstrucción social para la toma de decisiones, como condición inherente a la consolidación del proyecto de vida

Por su parte Montiel, Espitia y Guerra (2015), destacaron en su Proyecto Padres de familia aprendiendo con las TIC para ayudar a sus hijos en las tareas escolares, encontraron que estos presentan mayor motivación cuando trabajan de la mando con sus padres; más sin embargo también encontraron que los padres presentan dificultad en el uso de las herramientas tecnológicas y sus aplicaciones,

Estos antecedentes muestran la importancia del trabajo articulado entre la comunidad educativa y los padres de familia con el uso de las TIC, para ayudar y dar respuesta a las necesidades educativas de los estudiantes.

En la Institución Educativa Departamental María Inmaculada del Municipio de Pivijay Magdalena, es notoria la falta de conocimiento de los padres de familias y/o cuidadores en el uso de las TIC, evidenciándose en la poca colaboración, orientación y compromiso que les brindan a sus hijos entorno a las actividades escolares. Es de gran importancia resaltar, que la mayoría de los padres de familias y/o cuidadores de $5^{\circ}$ a y $5^{\circ} \mathrm{b}$ de la Institución Educativa Departamental María Inmaculada están caracterizados por falta de escolaridad, bajo nivel económico y disfuncionalidad en la estructura familiar; lo cual repercute en el comportamiento y rendimiento escolar del estudiante. Por lo tanto, es de gran interés emprender este proyecto investigativo debido a que contribuye en la calidad de vida de los padres de familias y/o cuidadores y estudiantes en los procesos vitales del ser humano como seres activos, innovadores y transformadores de una mejor sociedad. 


\section{Las TIC en el aprendizaje}

Partiendo del propósito de investigación se pueden relacionar los diferentes supuestos teóricos y conceptos que sustentan la importancia de trabajar en el contexto educativo con la participación de la familia. En este orden de ideas, se presentan los planteamiento de Picardo, Balmore y Escobar (2004), quienes en su proyecto estrategias pedagógicas en el ámbito educativo, resalta la importancia de implementar estrategias pedagógicas, que despierten el interés de los estudiantes por aprender; entendiendo como una estrategia pedagógica, es aquella donde las actividades se realizan, bajo unos parámetros o lineamientos previamente diseñados, con el fin de dar cumplimiento a objetivos de carácter educativo, es decir, constituye cualquier actividad o tarea planificada que busca fortalecer y facilitar la adquisición de aprendizajes, buscando el crecimiento pedagógico del estudiante.

Otro aporte significativo en relación a la implementación de las TIC en cl contexto educativo es el propuesto por las autoras Bautista, Martínez y Hiracheta (2014), quienes plantean el uso de herramientas tecnológicas, como los celulares, laptops, tablet, etc, como una estrategia didáctica para mejorar los resultados académico; pues estos otorgan gran destreza y facilitan el proceso de enseñanza aprendizaje, donde el estudiante y el docente las utilizan para generar distintos materiales que dinamicen e innoven la adquisición de capacidades, habilidades y aptitudes para su desarrollo humano y así alcanzar un mejor nivel educativo. Coinciden Talavera y Marín (2015), cuando plantean la necesidad de integrar los recursos tecnológicos a la mediación didáctica de forma tal que se fortalezca el aprendizaje significativo desde la socialización de nuevos contenidos didácticos.

Por su parte, Sánchez (2011), señala que los escenarios, educativos y sociales están mediados por el poder de la televisión, internet y la telefonía móvil, por su poder de influir en el mundo de la comunicación a distancia e instantánea, lo cual supera la mera transmisión de información, para convertirse en herramientas fundamentales para la diversión, el entretenimiento, la enseñanza, el aprendizaje y la exploración social.

Teniendo en cuenta que los escenarios actuales y futuros en la educación son cambiantes, no solo es necesario la participación de los padres en el contexto educativo, pues es fundamental, que los maestros se consoliden como estrategas, que se actualizan y portan al desarrollo tecnología desde el aula de clase, pues la formación de los maestros es clave para garantizar el aprovechamiento de las herramientas disponibles en la era digital. Es por ello, que es una necesidad u obligación que el docente debe tener la capacitación necesaria para que sea competente en el uso y apropiación de las TIC como herramientas pedagógicas (Pinto, Cortés, y Alfaro 2017).

Partiendo de esto, se pude afirmar que tanto los maestros como los padres, juegan un papel fundamental en el proceso de enseñanza aprendizaje, donde el acompañamiento debe ser permanente y continuo, esto aporta al proyecto investigativo estrategia pedagógica basada en las TIC orientada a padres de familia y/o cuidadores de los estudiantes de $5^{\circ}$ a y b de la Institución Educativa Departamental María Inmaculada Del Municipio De Pivijay Magdalena, ya que ratifica la importancia del acompañamiento de los padres tanto en la escuela como en el hogar, para ayudarlos con el cumplimiento de sus deberes escolares.

García y Mendia (2015), resaltan la importancia del acompañante como educador, el acompañante es una persona que contribuye con sus competencias, conocimientos y experiencia al crecimiento personal y social de las personas y grupos en el proceso educativo, pues aportan su crítica constructivista, su trayectoria y su empatía para estimular al grupo y al individuo a que construya su propio proyecto. 


\section{Metodología}

El abordaje metodológico del presente estudio se orientó desde el enfoque Cualitativo, donde Blasco y Pérez (2007) señalan que la investigación cualitativa aborda la realidad en su contexto, para sacar e interpretar fenómenos de acuerdo con las personas implicadas. A partir de esto, en la presente investigación se busca identificar el apoyo recibido por parte de los padres y cuidadores con respecto al manejo de las herramientas tecnológicas en la elaboración de actividades académicas de los hijos, en este sentido no se busca cuantificar el nivel de apoyo recibido, simplemente estudia la importancia o resultados del trabajo colaborativo entre padres e hijos con apoyo de las TIC.

El alcance de la investigación es descriptivo, porque desarrolla de modo sistemático las características de una situación o área de interés, además de los acontecimientos actuales de los padres de familias y/o cuidadores de la Institución Educativa María Inmaculada.

\section{Participantes}

Se tomó como unidad de análisis a 20 padres de familias y 9 cuidadores de los estudiantes del grado quinto A y quinto B, de la Institución Educativa Departamental María Inmaculada del Municipio de Pivijay Magdalena; cabe destacar, que los participantes dieron su consentimiento para poder divulgar los resultados.

A continuación, se presentan la selección de los participantes, los cueles fueron seleccionados mediante un muestreo intencional.

TABLA 1

Número de Participantes

\begin{tabular}{cccc}
\hline Padres de familia & Grado & Cuidador & Grado \\
\hline 18 & $5 \mathrm{~A}$ & 2 & $5 \mathrm{~A}$ \\
7 & $5 \mathrm{~B}$ & 2 & $5 \mathrm{~B}$ \\
\hline
\end{tabular}

Fuente: elaboración propia.

\section{Técnicas e instrumentos}

Para el presente estudio se seleccionó como técnicas de recolección de datos la entrevista, la cual según, Díaz, Torruco, Martínez \& Varela (2013), la definen como el conjunto de procedimientos mediante el cual el entrevistador emplea preguntas con el fin de conocer u obtener una información relevante, ajustada los objetivos que desea conocer de acuerdo al propósito de la investigación.

\section{Procedimiento}

Para la construcción del instrumento o entrevista inicialmente se partió de un proceso de observación diagnóstica con los padres de familia y cuidadores, realizando actividades pedagógicas apoyadas en TIC con los estudiantes; posteriormente se procedió a la construcción del instrumento incorporando contenidos de acuerdo con la problemática estudiada, y fue enviada a pares expertos para su respectiva validación, una vez validados los contenidos se procedió a elaborar y diseñar la entrevista tomando los aportes más significativos de la evaluación. Una vez se culminó el diseño de la entrevista se envió nuevamente a los expertos quienes iniciaron las correcciones y recomendaciones pertinentes. Finalmente elaborada la entrevista, se procede a realizar la aplicación.

TABLA 2

Formato de entrevista.

\begin{tabular}{cl}
\hline N. & \multicolumn{1}{c}{ Pregunta } \\
\hline 1 & $\begin{array}{l}\text { ¿Qué otros recursos considera usted son } \\
\text { importantes, para la orientación académica de } \\
\text { sus hijos? }\end{array}$ \\
2 & $\begin{array}{l}\text { ¿Usted tiene habilidad para el manejo de las } \\
\text { herramientas tecnológicas. SI o NO, por qué? }\end{array}$ \\
3 & $\begin{array}{l}\text { ¿Qué temas de interés son importantes para } \\
\text { orientar el aprendizaje de su hijo? }\end{array}$ \\
4 & $\begin{array}{l}\text { ¿En qué momento específico apoya usted a su } \\
\text { hijo respecto al proceso académico, explique } \\
\text { como lo hace? }\end{array}$ \\
& $\begin{array}{l}\text { ¿Cuáles son las asignaturas en la cual su hijo } \\
\text { presenta mayor dificultad, y usted como lo } \\
\text { orienta? }\end{array}$ \\
\hline
\end{tabular}

Fuente: elaboración propia. 


\section{Resultados}

A través de la aplicación del instrumento se detectaron factores determinantes en la adquisición de saberes de los estudiantes mediados por los padres, donde se evidencia la necesidad de conectar y orientar el sistema educativo al trabajo articulado con los padres de familia y cuidadores, con el fin de garantizar un aprendizaje eficaz, autónomo, ajustado a las exigencias tecnológicas y científicas que plante la educación actual, comprometido con el desarrollo ético y moral, aportando a la construcción de su entorno y comunidad académica. El instrumento se realizó y aplicó bajo la pregunta orientadora de la investigación, mediante la cual se busca determinar de qué manera la estrategia pedagógica basada en las tecnologías de la información y comunicación, orientada a padres de familia y/o cuidadores de los estudiantes $5^{\circ}$ a y $5^{\circ}$ b de la Institución Educativa Departamental María Inmaculada del Municipio de Pivijay Magdalena.

Continuación se presentan los principales resultados encontrados derivados de la aplicación de la entrevista de acuerdo a los objetivos planteados.

TABLA 3

Análisis cualitativo de resultados: Incidencia de las TIC desde la perspectiva de los padres y cuidadores

\begin{tabular}{|c|c|}
\hline Pregunta & Respuesta \\
\hline $\begin{array}{l}\text { Qué otros } \\
\text { recursos } \\
\text { considera } \\
\text { usted son } \\
\text { importantes, } \\
\text { para la } \\
\text { orientación } \\
\text { académica de } \\
\text { sus hijos? }\end{array}$ & $\begin{array}{l}\text { Materiales didácticos en cuestión } \\
\text { de matemáticas", "Internet", } \\
\text { "Computador", "Los libros, } \\
\text { "Implementos culturales". Respecto a } \\
\text { los cuidadores de los estudiantes } 5^{\mathrm{a}} \text { y } \\
\text { b; "Internet o la biblioteca }\end{array}$ \\
\hline
\end{tabular}

Partiendo de los resultados obtenidos se puede inferir que la mayoría de los padres de familias y/o cuidadores manifiestan que uno de los recursos utilizados para orientar a sus hijos en las actividades escolares es el internet y computador., en este sentido Martínez y Hiracheta (2014), plantean que el uso de material didáctico y las tecnologías de información y comunicación (TIC), son necesarias para mejorar el alcance académico, pues los estudiantes se motivan al manejar este tipo de tecnologías, lo que lleva a emplear nuevas estrategias innovadoras y dinámicas que faciliten la obtención de conocimientos, habilidades y aptitudes para que puedan alcanzar un mejor nivel educativo

De acuerdo con lo anterior se puede evidenciar

Si "He realizado curso", "Conozco lo básico de un computador", "Es una oportunidad para ayudar a mi hijo y así ver las noticias que se pueden

¿Usted tiene presentar en las redes sociales", "En habilidad para el manejo de las herramientas tecnológicas. SI NO, por qué? la secundaria me dieron lecciones de informática", "Me gustaría aprender un poco más". No "No hay en la Casa esas herramientas", "Yo nunca he manejado computador", "No tengo como capacitarme", "No tengo facilidad para internet", "El manejo de estas herramientas consiste en práctica y lastimosamente no tengo los medios para adquirir los (aparatos)". que la mayoría de los padres de familias y/o cuidadores no cuentan con las herramientas tecnológicas en sus hogares y no poseen el conocimiento para su manejo. Cabe resaltar que tal como señala, (Ramírez, 2010), las Tecnologías de la información y la comunicación (TIC) son herramientas útiles que permiten llevar a cabo prácticas y estrategias innovadoras que son de gran utilidad para fortalecer procesos de aprendizaje significativo en el contexto escolar. Además, se ha demostrado que las estrategias basadas en las TIC, como herramientas pedagógicas dentro y fuera del aula incrementan la iniciativa y motivación de los estudiantes en relación a la apropiación de conocimiento frente a sus compromisos escolares (Avendaño y Martínez, 2013). 
$\cdots$

\begin{tabular}{ll}
\hline Pregunta & Respuesta \\
\hline
\end{tabular}

"Aprender sobre La convivencia en el aula", "Ayudar hacer las actividades y ser responsable y explicarle en las

¿Qué tareas", "Manejar con mayor facilidad temas de el internet para ayudarlos cuando interés son lo necesiten", "A mí me gustaría importantes aprender sobre las redes sociales para para orientar el aprendizaje de su hijo?

¿En qué momento específico apoya usted a su hijo respecto al proceso académico, explique como lo hace?

\section{¿Cuáles}

son las asignaturas en la cual su hijo presenta mayor dificultad, y usted como lo orienta? enseñarle más a mis hijas". Respecto a los cuidadores; respondieron "Consultar en internet para apoyar a mis hijos", "A mí me gustaría aprender en las redes sociales ya que los niños están expuestos a muchos peligros", "Me gustaría aprender internet para enseñar".

Cuando no comprende una tarea le ayudo, explicándole como se hace sin hacerle la tarea y lo acompaño hasta que lo logre", "Cuando mi hijo tiene una tarea o trabajo les ayudo, a través del internet", "Ser responsable con las tareas en casa", "Ayudándolo con sus tareas en el momento que el necesite ayuda", "Busco una persona que lo sepa y que le ayude para que aprenda", "Lo ayudo hacer las tareas y lo oriento para que pueda estudiar mejor y lo hago en horas de la tarde", "Ayudando en las tareas acudiendo a las reuniones", "A mis hijo lo ayuda una profesora", "Yo lo apoyo siempre en todo".

"Lenguaje, motivándolo a leer, practicar escritura, prestando libro de su interés", "Ingles, nos ayudamos con el internet", "Matemática, le dificulta dividir y aprenderse la tabla de multiplicar", "Español, por la ortografía yo trato de ponerlo y corregirle siempre los errores", "Matemática, valores, sociales y naturales, haciéndole entender que él puede salir adelante con sus asignaturas", "Matemáticas, los hermanos les explica las divisiones y multiplicaciones", "a veces no quiere hacer las tareas y yo lo ayudo mucho y lo se orientar". Respecto a los cuidadores de los estudiantes $5^{\mathrm{a}}$ y b; respondieron "Mi hijo tiene dificultad en las matemáticas, ayudándolo en las divisiones”, “español”.
De acuerdo a los resultados es evidente la necesidad de los padres de familia y /o cuidadores de aprender sobre el uso y manejo de los equipos tecnológicos y en específico las redes sociales para el acompañamiento de los procesos escolares con la intención de mejorar el modelo didáctico que garantice una integración de las TIC en los procesos de enseñanza-aprendizaje, donde se hace necesaria a la participación de toda la comunidad educativa y en especial la familia del estudiante. Es importante tal como señala Martínez, Pagán, García \& Máiquez, (2013), que el uso y la implementación pedagógica de las TIC deben ir relacionadas con el avance y los cambios en el sistema educativo, en los que tenga en cuenta el carácter formativo del estudiante, en el que se fomente la relación escuela, TIC y familia, que sirva para desarrollar modelos que tengan en cuenta los canales de información entre maestros, directivos y familia.

Se puede inferir de lo anteriormente expuesto, el apoyo recibido por parte los padres y/o cuidadores cuando sus hijos necesitan de su acompañamiento. De acuerdo a esto Peña \& de Guzmán (2010), sostienen que al trabajar articuladamente la familia, el centro educativo, se fortalece una educación que promueve el pensamiento, la autonomía, la aceptación y el respeto de las diferencias, la imaginación y la crítica; capacidades que le permiten al estudiante resolver los problemas que surgen en la convivencia personal y grupal, a comprender y dar respuestas a los conflictos desde postura, critica, positiva, que promueva el cambio para el crecimiento como seres humanos y miembros de una sociedad.

De acuerdo a las respuestas dados por los padres de familia y cuidadores, las asignaturas en las que mayor presentan dificultades sus hijos se encuentran; matemática, valores y sociales; resaltando que los padres de familias y/o cuidadores le brinda su motivación para mejorar su rendimiento académico. En este sentido García y Mendía (2015) resaltan que el padre de familia o cuidador debe contribuir con sus competencias y bagaje experiencial, a la adquisición de nuevos saberes, a, mejorar en aquellas falencias, presentes al a nivel personal, social en el proceso educativo. Estos a través de práctica cotidiana y experiencia deben aportar su conocimiento y estimular al grupo y que sea autónomo en la construcción de su propio aprendizaje. 


\section{Discusión}

Las rápidas transformaciones sociales derivadas de la influencia de las nuevas tecnologías de la comunicación y la información; han marcado un cambio en el papel de la familia, asumiendo nuevos roles marcados por la adaptación, el trabajo y acompañamiento de los padres en las actividades académicas de los hijos mediadas por las TIC; en este sentido Martínez; Pérez y Solano (2011). Sostienen que en la actualidad, la implicación de los avances tecnológicos en el ámbito de las relaciones familiares ha tendido a modificar significativamente las formas de interacción de cada uno de sus miembros, su cotidianidad, sus modos de socialización y sobre todo, sus maneras de responder al medio.

A raíz de los resultados surge la necesidad de replantearse el ejercicio de la práctica pedagógica, implementando estrategias innovadoras que involucren a los padres de familia como entes fundamentales en la adquisición de conocimientos, como mediadores de conductas protectoras en cuanto al uso de las herramientas tecnológicas que posibiliten a nivel académico mayores y mejores resultados. Cabe considerar que tal como señala Martínez; Pérez y Solano (2011), que las personas o los contenidos a los que los hijos tienen acceso, en muchas ocasiones pueden ser riesgosos, por lo que se hace necesario el acompañamiento $\mathrm{y}$ supervisión de los padres en el manejo de las TIC; de la misma forma, el aumento del consumo tecnológico tiende a generar aislamiento social, ya que los tiempos que se dedican al medio audiovisual en muchas ocasiones tienden a generar conductas de aislamiento el deteriorando las relaciones sociales.

Se hace necesario lograr despertar un alto sentido de compromiso y responsabilidad de los padres y/o cuidadores sobre los procesos de aprendizaje de los estudiantes de la Institución Educativa Departamental María Inmaculada del Municipio de Pivijay-
Magdalena, aportando en gran medida a la búsqueda de sus tareas y compromisos a través de a internet, promedio de diferentes objetos electrónicos como computadores, tablets, celulares, etc. Teniendo en cuenta lo beneficioso que resulta el empleo de estrategias digitales y didácticas para aportar al crecimiento académico de los estudiantes.

Se logra constatar la importancia y alta incidencia que tienen las nuevas tecnologías de la información y la comunicación en el aprendizaje y en la forma en cómo interactúan los estudiantes con sus pares y superiores., tal como señalan , Marín, Inciarte, Hernández, \& Pitre (2017), que para que los futuros profesionales adquieran las competencias necesarias para aprovechar las ventajas ofrecidas por la innovación y las TIC, es necesario que las instituciones desarrollen estrategias de integración que se orienten a la consolidación de procesos pedagógicos y de aprendizaje, las cuales permitan tanto a los docentes como a los estudiantes y por tanto a los padres de familia comprender el alcance de las TIC en el contexto educativo; identificando la importancia de la innovación como eje transversal de todo proceso de desarrollo integral.

En conclusión se hace evidente la necesidad de implementar ambientes virtuales de aprendizaje, desde la institución como fuera de esta, en la que se realicen actividades compartidas entre padres y alumnos como una solución innovadora frente a las necesidad que presenta la educación de hoy; se plantea la estrategia de trabajar desde la cotidianidad con herramientas tecnológicas que facilitan el dinamismo de los espacios académicos, lo que a su vez repercute e impacta directamente en los interés y motivación de los estudiantes y, por tanto, con el acompañamiento adecuado por parte de los padres o cuidadores, lograr en el estudiante potencializar las competencias científicas, investigativas y tecnológicas, sumado a esto mejorar y fortalecer las relaciones fraternales entre padres e hijos. 


\section{Referencias}

Bautista, M., Martínez, A. y Hiracheta, R. (2014). El uso de material didáctico y las tecnologías de información y comunicación (TIC'S) para mejorar el alcance académico. Ciencia y tecnología, 14, 189-192.

Betancur, S., Carmona, L., Contreras, R., Karam, J., Maestre, N., Romero, Y. y Uribe, S. (2014). Videojuegos y TIC como estrategias pedagógicas: Formación para el uso seguro de internet. Cultura Educación y Sociedad, 5(1).

Blasco, J. y Pérez, J. (2007). Metodologías de investigación en las ciencias de la actividad física y el deporte: ampliando horizontes. España: Editorial Club Universitario.

García-Pérez, A. y Mendía, R. (2015). Acompañamiento educativo: el rol del educador en aprendizaje y servicio solidario. Revista de currículo y formación del profesorado, 19 (1), 42-58.

Díaz, L., Torruco, U.; Martínez, H. y Varela, M. (2013). La entrevista, recurso flexible y dinámico. Investigación en educación médica, 2(7), 162-167.

Hurtado, B., De la Hoz, Y. y De la Hoz, K. (2015). Implementación de estrategias pedagógicas dirigidas a los padres de familia para mejorar el rendimiento escolar de los niños y niñas de la escuela nueva frutier de santa Catalina [Tesis de pregrado]. Universidad de Cartagena, Cartagena. Recuperado de: http://190.242.62.234:8080/jspui/ handle/11227/2110.

Kliksberg, B. (2008). La familia en América Latina. Un tema decisivo. Conferencia inaugural del Congreso Internacional sobre Población, Vida y Desarrollo. Evidencias e interrogantes en los albores del siglo XX. Bogotá, D.C.: Universidad de La Sabana.
Marín, F., Inciarte, A., Hernández, H. y Pitre, R. (2017). Estrategias de las Instituciones de Educación Superior para la Integración de las Tecnología de la Información y la Comunicación y de la Innovación en los Procesos de Enseñanza. Un Estudio en el Distrito de Barranquilla, Colombia. Formación universitaria, 10(6), 29-38.

Martínez, M., Frías, W. y Solano, D. (2016). Impacto de los medios masivos de comunicación en la dinámica familiar. Cultura Educación y Sociedad, 2(1). 111-118.

Martínez, J., Pagán, F., García, A. y Máiquez, M. (2013). Las Tecnologías de la Información y la Comunicación en la relación familia-escuela. Revista Fuentes, (13), 173-192.

Millán, M. Gutiérrez, A. y Hernández, L. (2016). Concepciones sobre familia en adolescentes escolarizados en instituciones educativas distritales en Bogotá, Colombia. Cultura Educación Y Sociedad, 7(1), 23-38.

Montiel, M., Espitia, F. y Guerra, D. (2015). Padres de familia aprendiendo con las TIC para ayudar a sus hijos en las tareas escolares. [Tesis de especialización]. Fundaciòn Universitaria Los Libertadores, Canalete. Recuperado de http://repository.libertadores.edu.co/ bitstream/handle/11371/946/MontielCausilMarioManuel.pdf?sequence $=2$

Núñez, E. y Vergara, R., \& Bocanegra García, J. (2014). Sistema experto basado en lógica difusa tipo 1 para determinar el grado de riesgo de preeclampsia. INGE CUC, 10(1), 43-50. Recuperado de https://revistascientificas.cuc.edu.co/ ingecuc/article/view/341

Peña, E. y De Guzmán, P. (2010). Desafíos de la familia actual ante la escuela y las tecnologías de información y comunicación. Educatio Siglo XXI, 28(1). 41-68. 
Pinto, A., Cortés, O. y Alfaro, C. (2017). Hacia la transformación de la práctica docente: modelo espiral de competencias TICTACTEP. Pixel-Bit. Revista de $\mathrm{Me}$ dios y Educación, (51). 37-51.

Picardo, O., Balmore, R., y Escobar, J. (2004). Diccionario Enciclopédico de Ciencias de la Educación. San Salvador, El Salvador, C.A.: Centro de Investigación Educativa.

Ramírez, C. (2010), Las TICS en el Aula Granada: NNTT.

Romero-Acosta, K. y Contreras, E. (2015). Revisión teórica sobre el post-conflicto: una oportunidad para empoderar a mujeres víctimas de desplazamiento. $\mathrm{Cul}$ tura Educación y Sociedad, 6(1). Recuperado de https://revistascientificas.cuc.edu.co/culturaeducacionysocie$\mathrm{dad} / \mathrm{article/view/757}$
Sánchez, E. (s.f.). Las TIC como medio de investigación y evaluación en un estudio sobre estilos de vida. Málaga. Recuperado de1: http://gtea.uma.es/congresos/ $\mathrm{CDROM} /$ comunicaciones/carpeta1/Comunicacion_sanchezvega_Elena-1.pdf

Strack, J., Suárez, J., Di Mauro, G. y Jacob, S. (2014). Impacto de la iluminación residencial eficiente en la calidad de la energía de una red de distribución. INGE CUC, 10(2), 9 - 19. Recuperado de https://revistascientificas.cuc.edu.co/index.php/ingecuc/article/view/485

Talavera, R. y Marín, F. (2015). Recursos Tecnológicos e Integración de las Ciencias como Herramienta Didáctica. Revista de Ciencias Sociales (RCS) 21(2). 337-346

Vargas, G. (2003). Filosofía, Pedagogía, Tecnología. Investigaciones de Epistemología de la Pedagogía y Filosofía de la Educación. Bogotá, D.C.: Alejandría Libros. Recuperado de http://www.pedagogica. edu.co/storage/folios/artículos/ folios17_12rese.pdf 